\title{
Genome-wide identification, evolution and expression analysis of the aspartic protease gene family during rapid growth of moso bamboo (Phyllostachys edulis) shoots
}

Xiaqin Wang ${ }^{1,2,3}$, Xinyang Yan ${ }^{1,2}$, Shubin $\mathrm{Li}^{1}$, Yun Jing ${ }^{1}$, Lianfeng $\mathrm{Gu}^{1}$, Shuangquan Zou ${ }^{1,2}$, Jin Zhang ${ }^{3^{*}}$ and Bobin Liu ${ }^{1,2^{*}}$ (D)

\begin{abstract}
Background: Aspartic proteases (APs) are a class of aspartic peptidases belonging to nine proteolytic enzyme families whose members are widely distributed in biological organisms. APs play essential functions during plant development and environmental adaptation. However, there are few reports about APs in fast-growing moso bamboo.

Result: In this study, we identified a total of 129 AP proteins (PhAPs) encoded by the moso bamboo genome. Phylogenetic and gene structure analyses showed that these 129 PhAPs could be divided into three categories (categories A, B and C). The PhAP gene family in moso bamboo may have undergone gene expansion, especially the members of categories $A$ and $B$, although homologs of some members in category $C$ have been lost. The chromosomal location of PhAPs suggested that segmental and tandem duplication events were critical for PhAP gene expansion. Promoter analysis revealed that PhAPs in moso bamboo may be involved in plant development and responses to environmental stress. Furthermore, PhAPs showed tissue-specific expression patterns and may play important roles in rapid growth, including programmed cell death, cell division and elongation, by integrating environmental signals such as light and gibberellin signals.

Conclusion: Comprehensive analysis of the AP gene family in moso bamboo suggests that PhAPs have experienced gene expansion that is distinct from that in rice and may play an important role in moso bamboo organ development and rapid growth. Our results provide a direction and lay a foundation for further analysis of plant AP genes to clarify their function during rapid growth.
\end{abstract}

Keywords: Aspartic protease, Moso bamboo, Programmed cell death, Rapid growth

\footnotetext{
* Correspondence: zhangi@zafu.edu.cn; liubobin@fafu.edu.cn

${ }^{3}$ State Key Laboratory of Subtropical Silviculture, School of Forestry and Biotechnology, Zhejiang A\&F University, Zhejiang 311300, Hangzhou, China

${ }^{1}$ College of Forestry, Fujian Agriculture and Forestry University, Fuzhou 350002, China

Full list of author information is available at the end of the article
}

(c) The Author(s). 2021 Open Access This article is licensed under a Creative Commons Attribution 4.0 International License, which permits use, sharing, adaptation, distribution and reproduction in any medium or format, as long as you give appropriate credit to the original author(s) and the source, provide a link to the Creative Commons licence, and indicate if changes were made. The images or other third party material in this article are included in the article's Creative Commons licence, unless indicated otherwise in a credit line to the material. If material is not included in the article's Creative Commons licence and your intended use is not permitted by statutory regulation or exceeds the permitted use, you will need to obtain permission directly from the copyright holder. To view a copy of this licence, visit http://creativecommons.org/licenses/by/4.0/ The Creative Commons Public Domain Dedication waiver (http://creativecommons.org/publicdomain/zero/1.0/) applies to the data made available in this article, unless otherwise stated in a credit line to the data. 


\section{Background}

Aspartic proteinases (APs; Enzyme Commission 3.4.23) are proteolytic enzymes and play important roles in protein maturation and degradation $[1,2]$. The majority of APs have two conserved motifs with catalytic activity: an Asp-Thr-Gly (DTG) motif and an Asp-Ser-Gly (DSG) motif $[3,4]$. APs are widely distributed among microbes, mammals and plants $[3,5]$ and are divided into 16 subfamilies based on their tertiary structure and evolutionary relationships $[4,6]$. APs are involved in many important biological processes that are involved in development, nutrition, pathogenesis, disease and so on and have potential for commercial application $[7,8]$.

Most plant APs are grouped into the A1 family and exhibit the two basic features of A1 family members: one features is that they are active under acidic conditions, and the other is that their catalytic activity can be specifically inhibited by pepstatin A [1,9]. Since the 1980s, plant APs have been purified via pepstatin Aagarose columns and detected in various plant species $[3,4,10]$. Plant APs can be classified into three categories: typical APs, nucellin-like APs and atypical APs [1, 9]. Typical APs contain a plant-specific insert (PSI) similar to that of saposin-like proteins, but it is removed during protein maturation $[1,2]$. Nucellin-like APs are similar to nucellins in barley ovules [11]. The characteristics of atypical APs are intermediate between those of typical and nucellin-like APs $[9,12]$. Pepstatin A activity has been detected in immature, mature, and germinated seeds in wheat, and the expression pattern showed a role of APs in regulating protein degradation [13, 14]. Plant APs are also considered to be responsible for protein processing and degradation, such as plant senescence, programmed cell death (PCD), reproduction, and stress responses $[2,15-20]$, which are critical for plant development. With the development of DNA sequencing technology, members of plant AP gene families have been identified in Arabidopsis [9], rice [12], grape [21], and poplar [22], revealing gene expansion and functional diversity [12, 22].

The function of plant APs has been determined primarily in seeds, including dormant seeds and different parts of seeds [10, 14, 23, 24]. It was proposed that, during seed development, plant APs are involved in seed storage protein processing on the basis of the $2 \mathrm{~S}$ albumin process in vitro and colocalization with proteins in the plant body [25]. During seed germination, plant APs are considered to be involved in seed storage protein degradation [26-28]. Recently, Arabidopsis ASPARTIC PROTEASE IN GUARD CELL 1 (ASPG1) was reported to promote seed germination by accelerating the breakdown of seed storage proteins [28]. In addition to their involvement in seed development and germination, APs participate in the degradation of insect proteins, allowing carnivorous plants to obtain nitrogen from those sources $[15,29]$. Plant APs also play roles in the response to biotic and abiotic stresses. ASPG1 is abscisic acid (ABA) inducible, and Arabidopsis plants overexpressing this gene had in increased ability to resist drought stress because of the participation of the transgene in ABA-dependent responsiveness [17]. Constitutive Disease Resistance 1 (CDR1), an atypical plant aspartic proteinase, exhibits the ability to induce systemic defense responses against bacterial and fungal pathogens in rice and Arabidopsis [20, 30, 31]. Ectopic expression of $V q A P 13$, a grape aspartic protease gene, can afford powdery mildew resistance but reduces Botrytis cinerea resistance by regulating the salicylic acid and MeJA signaling pathways [19]. Plant APs also play roles in plant development, such as reproduction and lateral root formation. OsAP65 has been proposed to be involved in biosynthesis of compounds that are essential to pollen germination and pollen tube growth in rice [32]. Two novel AtAPs in Arabidopsis (A36 and A39) have been speculated to participate in gametogenesis and pollen guidance [18]. Recently, an atypical aspartic protease, Atypical Aspartic Protease in Roots 1 (ASPR1), was determined to suppress primary root growth and lateral root development [33]. Altogether, plant APs are important proteins that are involved in various aspects of plant development and responses to environmental changes.

Some plant APs also play an important role in regulating PCD. In barley, a gene encoding an aspartic protease-like protein ('nucellin') was highly expressed after pollination, which was synchronized to nuclear cell degeneration characteristic of PCD [11]. Phytepsin, a vacuolar aspartic proteinase that is a plant homolog of cathepsin D and mediates PCD in barley, is highly expressed during the active autolysis of the root cap and in tracheary elements and sieve cells [34]. In rice, the transcripts of OsAP25 and OsAP37 in anthers are activated by ETERNAL TAPETUM 1 (EAT1) to regulate PCD in tapetal cells [35]. In Arabidopsis, PROMOTION OF CELL SURVIVAL 1 (PCS1) encodes an aspartic protease, and compared with wild type, loss-of-function mutants experience gametophyte degeneration and cell death of developing embryos [36]. AP proteins have also been identified in the plant cell wall, and cis-elements related to secondary cell wall (SCW) thickening and PCD, such as SNBE, TERE, and SMRE, were discovered upstream of partial AP genes from poplar, strongly suggesting that APs play important roles in SCW and PCD [37-41]. To date, there are many reports on plant AP function in model plant species such as Arabidopsis and rice. However, the function of APs in rapid-growing plant species such as bamboo is still unclear.

Bamboo is a member of the Gramineae family, is widely distributed worldwide and is a rapid-growing plant species. Bamboo forests can provide young 
bamboo shoots for food, fibrous raw material, building materials, raw materials for furniture and crafts and so on within a short time [42]. In addition to its economic benefits, bamboo also has important ecological functions, such as the ability to restore degraded landscapes and combat global climate change $[42,43]$. The moso bamboo (Phyllostachys edulis) planting area is approximately 3.27 million ha and constitutes most of the bamboo forest region in China [43]. Rapid growth of moso bamboo occurs after the young bamboo shoots are covered with a shell and emerge from the ground. PCD was revealed to occur in pith cavity formation during rapid bamboo growth [44]. During the bamboo rapid-growth stage, cell division gradually decreases, while cell elongation and secondary cell wall thickening also occur $[45,46]$. Therefore, PCD and SCW formation are important biological events during rapid growth of moso bamboo. Members of the NAC, MYB and LAC gene families have been identified as being associated with SCW in moso bamboo $[47,48]$. The MYB gene family has specifically been reported to be involved in environmental responses [49]. In addition to rapid growth, the flowering pathway $[50,51]$ and sucrose synthase [52] have also been widely studied in bamboo. Recently, a chromosome-level de novo genome assembly of moso bamboo was provided, which, compared with the previous version, was obviously improved in terms of the assembly data and quality of the whole-genome sequencing assembly $[43,53]$. The release of new bamboo genomic data allows us to perform genome-wide gene functional analyses in moso bamboo.

Here, we identified a total of $129 \mathrm{PhAP}$ proteins that contain a conserved Asp domain from the moso bamboo genome. Phylogenetic analysis revealed that PhAP genes might have experienced gene expansion via segmental and tandem duplication. Gene structure and motifs indicated that the motifs of PhAPs were conserved, although the gene structure has changed throughout evolutionary history. Expression pattern analysis showed that PhAPs exhibited tissue-specific expression patterns, and several sets of PhAPs may play important roles during moso bamboo rapid growth. Our study provides a strong foundation for further research on the potential function of these proteins in bamboo development and an improved understanding of the AP gene family in fast-growing nontimber forest species.

\section{Results}

Genome-wide identification of AP genes from the moso bamboo genome

After two rounds of moso bamboo genome searching via HMMER v3 (the details of which are in the materials and methods), a total of 129 Asp family proteins with a conserved Asp domain were analyzed via the NCBICDD and Pfam database (Fig. 1 and Table S1). Among these Asp proteins, 102 had two catalytic sequence motifs, Asp-Thr-Gly (DTG) and Asp-Ser-Gly (DSG), which are typical features of aspartic proteases; however, 18 proteins contained one catalytic motif, and nine proteins had no motif (Fig. 1 and Table S1). Moso bamboo Asp genes were named based on their relationships with homologous genes in rice and are listed in Table S1. Other information on the members of the Asp gene family, including their chromosomal localization, CDS, amino acid residue sequence, corresponding protein length, corresponding protein molecular weight, and corresponding protein isoelectric point, is also listed in Table S1.

Phylogenetic relationships among the 129 moso bamboo Asp proteins were determined using an IQ-TREE procedure [54]. The 129 moso bamboo Asp proteins fell into three distinct categories (pink, blue and purple clades) and were termed categories A, B and C, respectively (Fig. 1). From the predicted protein domain, we found that all PhAPs contained one Asp domain of variable length (Fig. 1). There were 16 moso bamboo category A PhAP members, eight of which contained signal peptides, and the Asp domain consisted of the Taxi_N and PSI domains (including SapB_1 and SapB_2) with two catalytic motifs (Fig. 1 and Table S1). However, there were no signal peptides or PSI domains and/or a lack or partial lack of catalytic motifs in the other eight category A PhAPs (Fig. 1 and Table S1). Categories B and $C$ had 26 and 87 members, respectively, that contained the full-length Asp domain consisting of Taxi_N and Taxi_ C, except for PhAP7.4, PhAP31.3, PhAP7.2, PhAP87.1, PhAP4.1, PhAP50.3, PhAP27.2 and PhAP40.2 (Fig. 1). Less than half of the category $\mathrm{B} P h A P$ s are nucellin-like APs containing catalytic sites (Fig. 1 and Table S1), which is similar to that which occurs rice [12]. Category C, composed of atypical aspartic proteases, was the largest category (Fig. 1). Most category B and C members contained a signal peptide, and it was notable that there were signal peptides and transmembrane domains located in the $\mathrm{N}$ - and C-termini, respectively, of nine category B AP proteins (Fig. 1).

Phylogenetic analysis of APs from moso bamboo and rice To investigate the evolutionary relationship of the PhAP family, a phylogenetic tree was constructed using $129 \mathrm{PhAP}$ and 92 OsAP full-length amino acid residue sequences (Table S1 and Table S2). Both PhAPs and OsAPs were classed into three categories, as previously reported in Arabidopsis [9], rice [12], grape [21] and poplar [22]. Category A contained 16 PhAPs together with seven OsAPs; these proteins could be classified into seven subclades based on their relationships with their rice homologous proteins (Fig. 2). There was at least one PhAP homolog in each subclade but no homolog of OsAP6 (Fig. 2). The moso bamboo genome encoded eight PhAP88 genes and only one homolog in rice, which meant that AP88 underwent gene expansion in moso bamboo (Fig. 2). 25 PhAPs and 15 OsAPs 


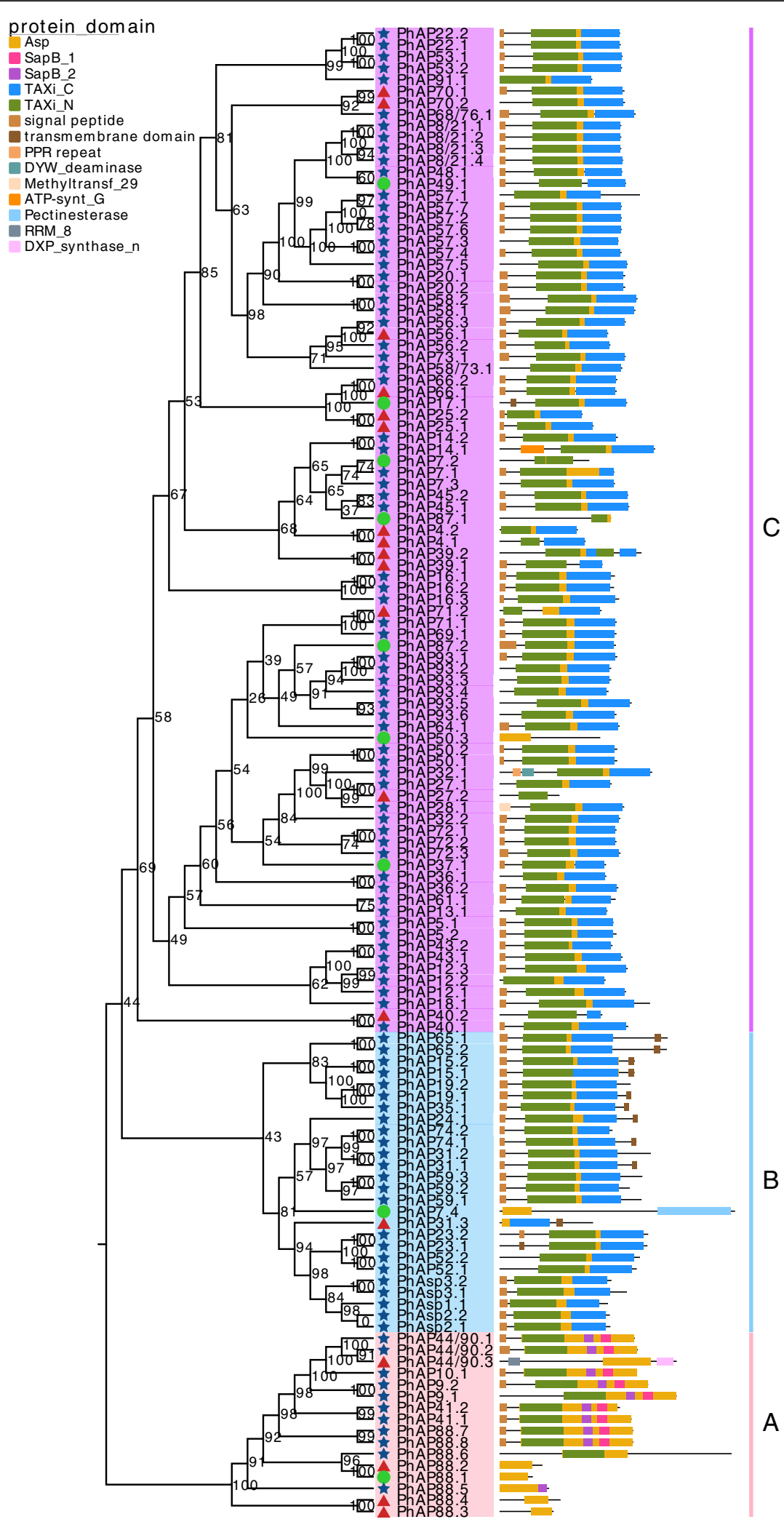

Fig. 1 (See legend on next page.) 
(See figure on previous page.)

Fig. 1 Phylogenetic relationships and protein domain diagram of moso bamboo aspartic proteinases. The left part shows the phylogenetic relationships of 129 APs from moso bamboo. Categories A, B And C are shaded in pink, blue and purple, respectively. The blue stars, red triangles and green circles represent APs containing 2, 1 and 0 catalytic sequences, respectively. Bootstrap are shown close to the branch nodes. The right part shows the protein domain, and the caption is shown in the upper left corner

were classified into category B, which could be further divided into 13 subclades (Fig. 2). Each subclade contained at least one PhAP homolog in moso bamboo (Fig. 2). Category $\mathrm{C}$ contained 87 PhAPs and 70 OsAPs. There was at least one PhAP homolog, and PhAP57 and PhAP93 exhibited evidence of gene expansion in moso bamboo (Fig. 2).
There were no homologous genes of OsAP77-87 in moso bamboo, indicating that the homologs in bamboo were lost during evolutionary history (Fig. 2). Altogether, these results showed that the PhAP gene family in moso bamboo underwent specific evolutionary events after the divergence of bamboo and rice.

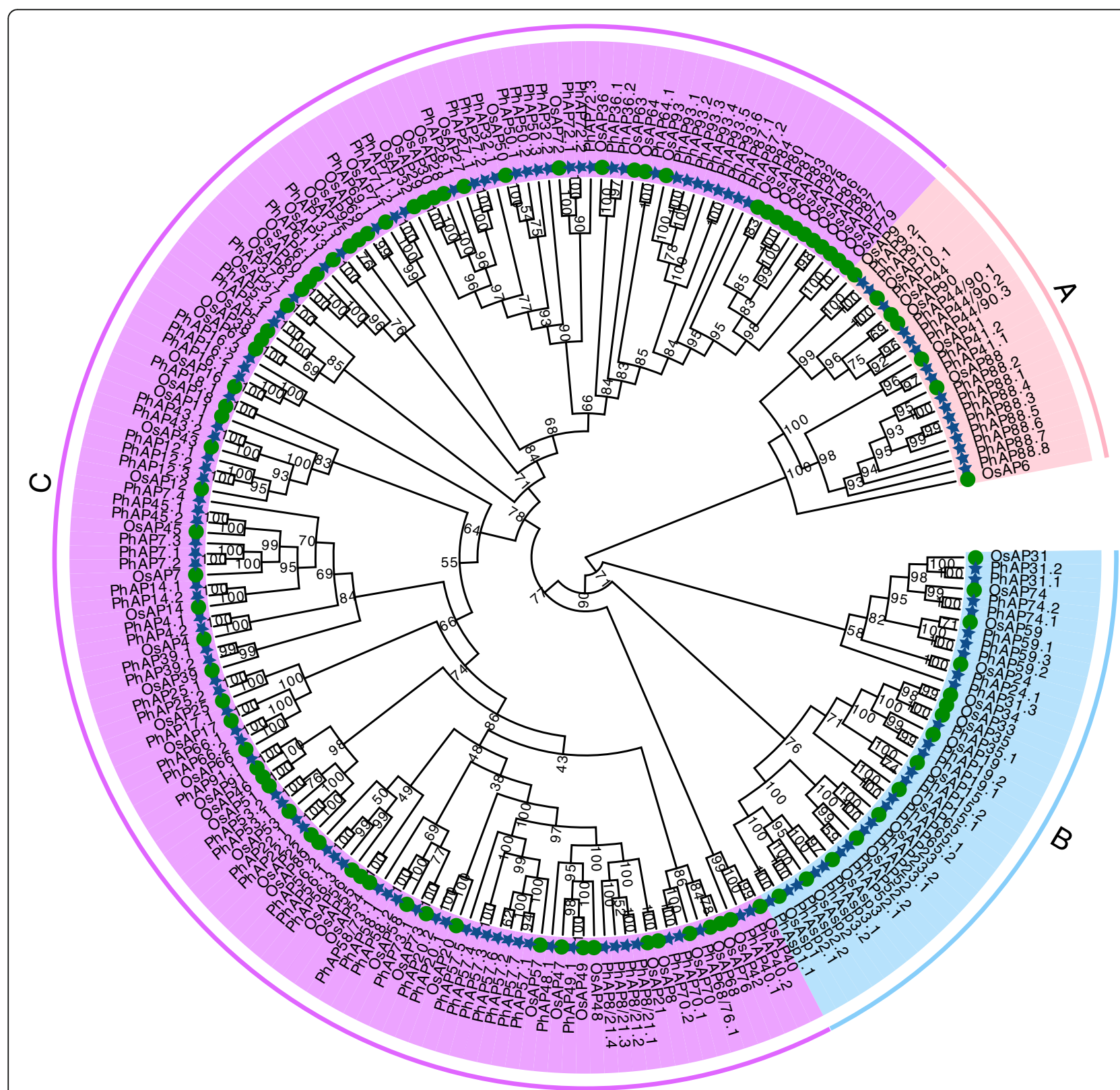

Fig. 2 Phylogenetic tree of moso bamboo and rice aspartic proteinases. Categories A, B and C are shaded pink, blue and purple, respectively. The blue stars and green circles represent moso bamboo APs and rice APs, respectively. The bootstrap percentages are shown close to the branch nodes 
Chromosomal location and gene duplication events of PhAPs We mapped the PhAPs onto chromosomes to examine the PhAP distribution on the moso bamboo chromosomes. Among the 129 PhAP genes, 124 were located on 21 out of 24 moso bamboo chromosomes, while the other five PhAPs were located on scaffolds (Fig. 3). Figure 3 shows that the chromosomal distribution of the PhAPs was nonrandom but was scattered and uneven. Fourteen PhAPs located on chromosome 6 contained the maximum number of PhAP genes; 13 PhAP genes were on chromosome $8 ; 12 \mathrm{PhAPs}$ were on chromosome 14; and chromosomes 2, 5, and 11 had only one PhAP gene (Fig. 3). There was no PhAP gene located on chromosome 1, 19, or 22 (Fig. 4). Segmental and tandem duplications are considered to be the main reasons leading to gene family expansion in plants. As shown in Fig. 3, some PhAP genes (PhAP8/21.1 and PhAP8/ 21.2; PhAP57.2 and PhAP57.3; and PhAP57.4,

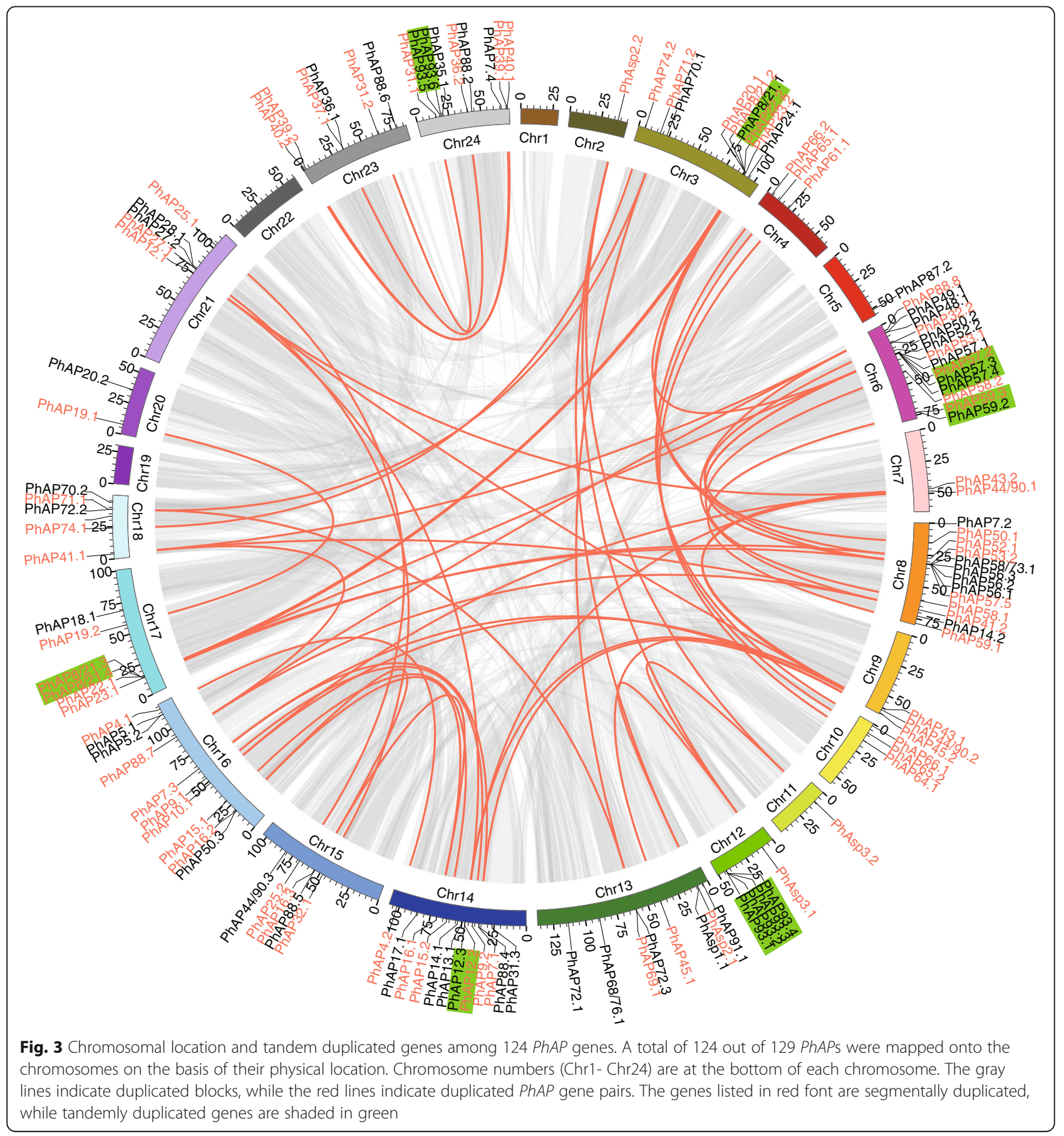




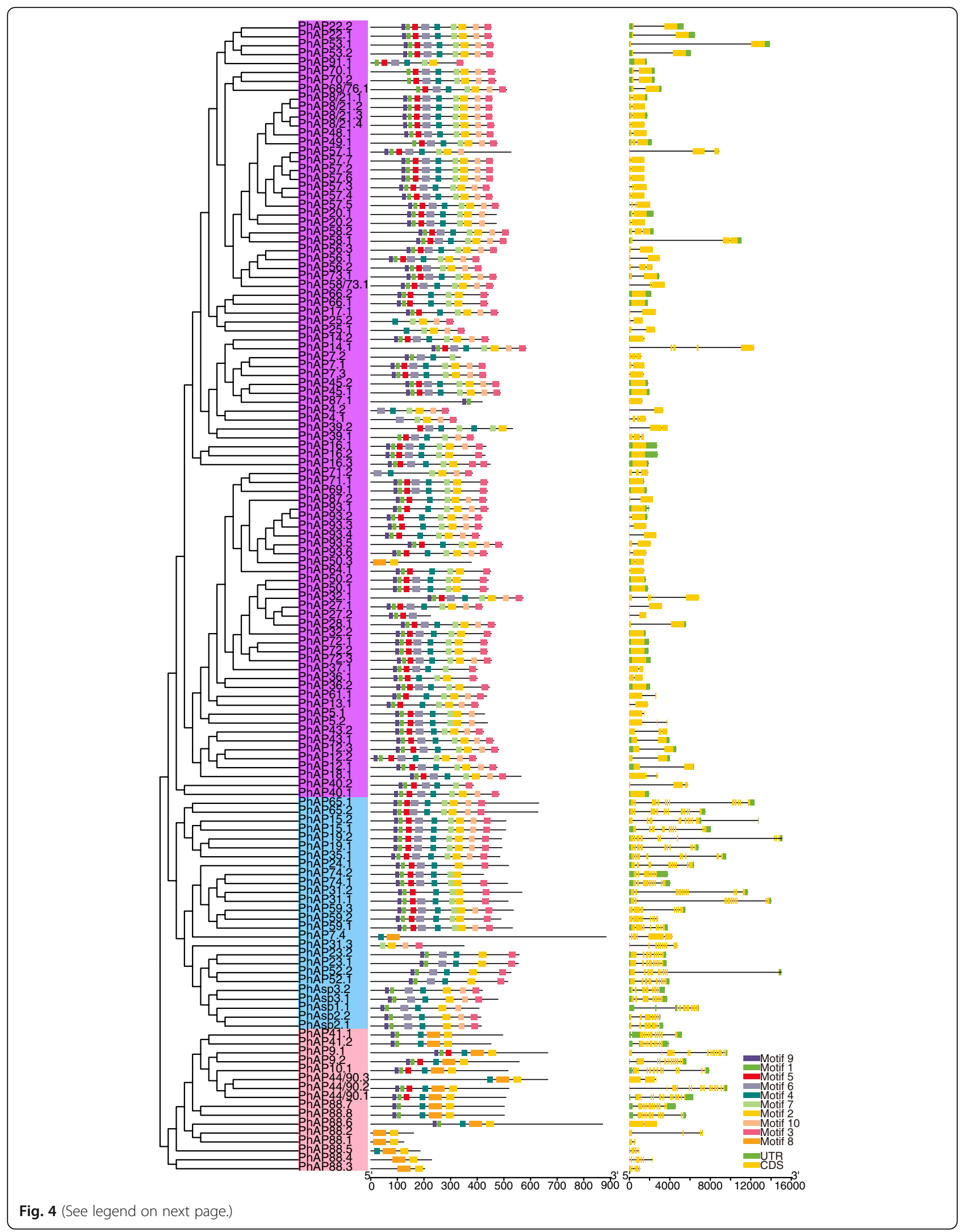


(See figure on previous page.)

Fig. 4 Phylogenetic tree of PhAPs as well as protein motifs and gene structure of corresponding PhAP genes from moso bamboo. The left panel shows the phylogenetic tree of PhAPs, as shown in Fig. 1. The middle panel shows the conserved protein motifs and their distribution. The boxes with different colors represent the conserved motifs listed in Fig. 5. The right panel shows the gene structure. The yellow boxes represent exons, the gray lines represent introns, and the green boxes indicate untranslated regions

PhAP93.1 and PhAP93.2 as well as PhAP93.3 and PhAP93.4; PhAP69.1 and PhAP72.3; PhAP8/21.3 and PhAP8/21.4; and PhAP93.5 and PhAP93.6) were adjacent to others and located sequentially in tandem on chromosomes $3,6,12,14,17$ and 24, suggesting that these genes might have expanded via tandem duplication (Fig. 3). In addition to the tandem duplication, we also found that 73 PhAPs were located in segmental duplication blocks (Fig. 3). Furthermore, approximate divergence dates and the $K \mathrm{a} / K \mathrm{~s}$ ratio of duplication pairs were estimated (Table S3). The results showed that duplication events may have taken place from 63.41 million years ago (Mya) to $0.2 \mathrm{Mya}$. The $\mathrm{Ka} / \mathrm{Ks}$ of the duplication pairs were $<1.0$ except for PhAP8/ 21.3 and PhAP8/21.4, PhAP57.3 and PhAP57.4, and PhAP93.1 and PhAP93.2, suggesting that most duplication pairs underwent purifying selection. These results indicated that most of the PhAP genes arose from tandem and segmental duplications, the processes of which play a very important role in the expansion of the PhAP gene family.

\section{Analysis of PhAP conserved motifs and gene structure}

The distribution of conserved protein motifs and gene structure are considered to play an important role in gene family evolution. First, we analyzed the conserved motifs of the PhAP proteins and their distribution by the MEME online tool. The conserved motifs and their distribution are shown in Fig. 4, while the corresponding logos are shown in Fig. 5. The conserved motif number in each of the PhAPs ranged from two to nine (Fig. 4). Motif 1 and motif 2 had the catalytic sequence motifs (Fig. 5) that were most conserved in the three categories and were present in nearly all members of PhAPs together with motif 4 and motif 9 (Fig. 4). Category A proteins had five or six conserved motifs, of which motif 8 was category A specific, with the exception of PhAP88.1-5 (Fig. 4). Motifs 3, 6, 7 and 10 were specific motifs of categories B and C (Fig. 4). Category B could be divided into two subclades based on the conserved motif distribution: the proteins in one clade had seven motifs, and the others had six motifs, except for PhAP7.4 and PhAP31.3 (Fig. 4). Like PhAP22s, the atypical category $\mathrm{C}$ members contained eight to nine motifs, but 18 members in category $\mathrm{C}$ had fewer than eight motifs because of the loss of some conserved motifs (Fig. 4). These results showed that the motifs were conserved in each category, although some PhAP members lost several motifs.

We further analyzed the exon/intron structure of the 129 PhAP genes (Fig. 4). PhAPs from different categories had distinct gene structures, which included exon/intron numbers, length and arrangement (Fig. 4). The gene structure of the category A genes was diverse (Fig. 4). PhAP88.7 and PhAP88.8 had 11 exons with similar exon numbers and distributions, whereas other homologs had two to eight exons of various lengths (Fig. 4); PhAP88.1PhAP88.8 were the closest homologs of OsPhAP88 (Fig. 2). The other homologs in category A, such as PhAP44/ 90s, PhAP9s and PhAP41s, also had different gene structures (Fig. 4). The gene structure of members of category B was conserved because the close homologs had similar exon/intron structures, although there were different exon numbers and distributions between the two branches of category B (Fig. 4). The number of exons in category $\mathrm{C}$ was at most five, which was different from the numbers in Populus and grape [21, 22], and most of the closest homologs, such as PhAP57s, $-14 s,-7 s$, and $-4 s$, on exhibited different gene structures (Fig. 4). These results indicated that the gene structure of PhAPs changed throughout evolutionary history.

\section{Cis-element analysis of PhAP family genes}

Cis-elements are located in the promoter region of target genes and interact with transcription factors to trigger target gene expression. We herein filtered ciselements of upstream sequences of the $129 \mathrm{PhAP}$ genes (Fig. 6). It was very clear that the most abundant cis-elements located in the promoter region of PhAPs were MYB-related elements and light-responsive elements (Fig. 6). Nearly all PhAPs contained an average of seven MYB-related elements and nine light-responsive elements (Fig. 6). Forty-one light-responsive elements and $18 \mathrm{MYB}-$ related elements existed in the promoter regions of PhAP40.2 and PhAP65.2, respectively (Fig. 6). The second most abundant cis-elements were MeJA-responsive and abscisic acid-responsive elements, MYC-related elements and anaerobic-inductive elements (Fig. 6). In addition, 89 PhAPs had several anaerobic-inductive elements, 66 PhAPs contained one to four metabolic regulatory cis-elements, and 61 PhAPs contained relatively few cis-elements that were auxin-responsive elements and gibberellinresponsive elements (Fig. 6). A portion of the PhAP upstream regions contained a small number of meristemrelated cis-elements (59 PhAPs), ethylene-responsive 


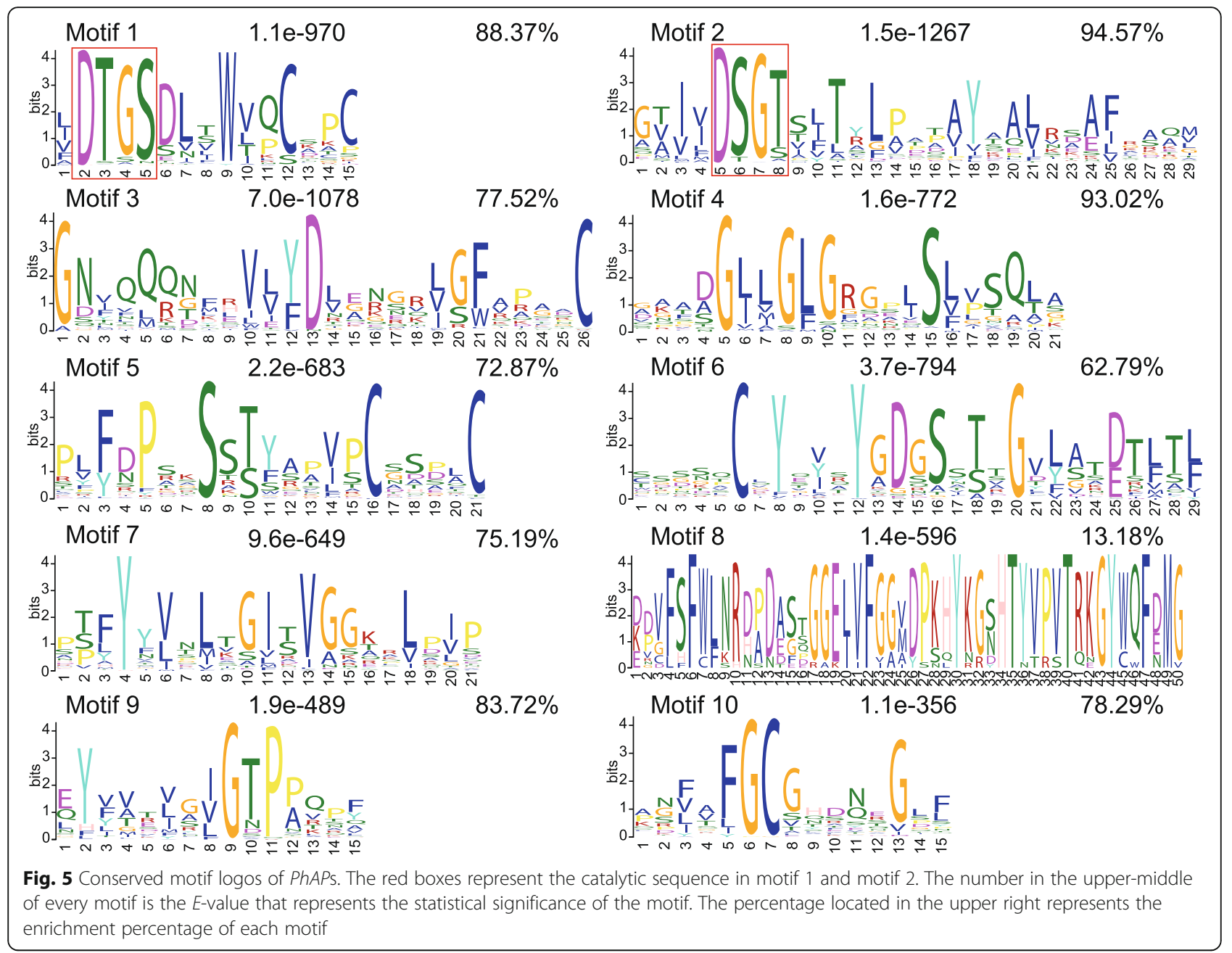

elements (57 PhAPs) and salicylic acid-responsive elements (42 PhAPs). Environmental stress-related cis-elements, such as drought-inducible elements, lowtemperature-responsive elements, defense and stressresponsive elements, and wound-responsive elements, were also identified in some PhAP genes (Fig. 6). No ciselement was identified from PhAP57.1, - 57.7, - 57.2, 57.6 or -88.3 (Fig. 6). These results suggested that PhAPs may be widely involved in development and responses to environmental changes in moso bamboo.

Because SCW formation and PCD have been reported to occur during the moso bamboo rapid-growth stage, PhAP genes may be involved with key transcription factors that regulate SCW and PCD. We further scanned 10 cis-elements previously discovered to participate in SCW formation and PCD by MEME scanning $[22,55]$. The results are shown in Figure S1. A total of 83\% (107 out of 129 PhAPs) of PhAPs had at least one cis-element related to SCW formation and PCD. There were 64 and 50 PhAPs containing SMRE1 and SMRE3 cis-elements, respectively, and 27 PhAPs had ACIII and SMRE5 cis- elements. Seventy PhAPs had SMRE2 cis-elements. SMREs and ACIII are located in the promoter regions of secondary wall biosynthetic genes and are responsible for SCW [56]. Thirty-five PhAPs have ACII and TERE cis-elements. Similarly, 31 PhAPs had SNBE cis-elements. TERE and SNBE are critical cis-elements responsible for PCD and SCW during tracheary [37] and vessel [38] element formation, respectively. Sixteen PhAPs had s ACI and M46RE. The cis-element M46RE, which is recognized by MYB46, is also involved in SCW [57]. SCW - and PCD-related cis-elements were also found upstream of PhAP57.1, - 57.2 and - 57.3 (Figure S1). To date, methyl jasmonate (MeJA)-, gibberellin (GA)-, ABA-, and ethylene-related as well as MYB transcription factors have been reported to be involved in PCD [5860]. Hence, these results suggested that PhAPs may be potentially involved in SCW and PCD processes.

Expression patterns of PtAP genes in moso bamboo tissues To reveal the potential function of $P h A P \mathrm{~s}$, we analyzed the expression pattern of PhAPs in various tissues, 


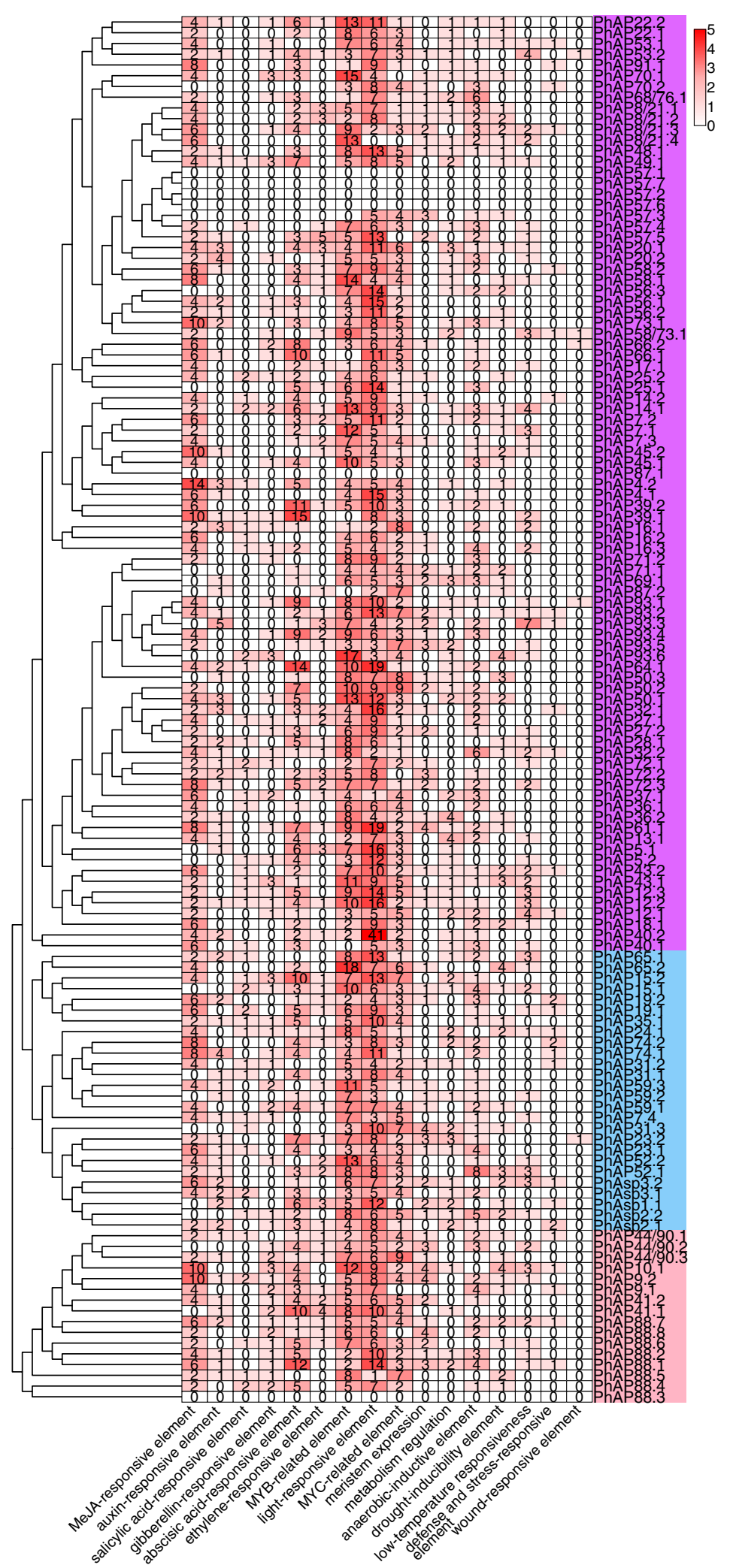

Fig. 6 Cis-element analysis of the promoter region of PhAPs. The cis-elements located in the 1500 bp promoter sequence (upstream of the start codon) of all PhAPs were analyzed via the PlantCARE website. The number of corresponding cis-elements was used for the heatmap construction 
including the roots, different portions of stems, leaf blades, leaf sheaths, buds, and rhizomes (Fig. 7), the data of which were obtained from publicly available RNA-seq data [43]. To check whether the RNA-seq data were reproducible, we randomly selected seven PhAPs and validated their expression patterns via RT-qPCR in different tissues of moso bamboo, including the top shoots, middle shoots, low shoots, leaves, sheaths, and roots. The results showed that the expression patterns of the seven PhAPs were consistent with the RNA-seq results (Figure S2). Figure 7 showed that the expression pattern of PhAPs were tissue preferences. The percentage of highly expressed $P h A P \mathrm{~s}$ in each tissue is summarized in Figure S3A. On the basis of their expression patterns, the $P h A P$ s were clustered into two main classes: class I and class II (Fig. 7). Almost all PhAPs showed transcript abundance in each tissue except class II $\zeta$, which included PhAP8/21.4, - 57.1, - 57.2, - 57.4, - 57.5, - 57.6, - 57.7, - 56.1, - 56.3, - 58/73.1, - 71.2, - 87.2, - 93.4, - 93.6, 27.2, -7.4 and -88.2 . Class I showed a high expression levels in the stems, although some members were highly expressed in the leaves or rhizomes (Fig. 7). In contrast, class II genes were nearly absent from the stems and showed various expression in the other above mentioned tissues (Fig. 7). Class II could be further divided into seven subclasses whose members exhibited preferential transcriptional abundance in the rhizomes (II $\alpha)$; leaves (II $\beta)$; roots (II $\gamma)$; buds (II $\delta$ ); roots, leaves and bud (IIE); and leaves and buds (II $\eta$ ) (Fig. 7). No mRNA expression was detected from class II $\zeta$ members, which included 17 PhAPs from the abovementioned tissues (Fig. 7). Some $P h A P$ s showed a highly specific expression pattern; for example, four PhAPs (PhAP91.1, - 16.3, - 72.2 and - 9.1) were mainly expressed in new roots with lateral roots, and four PhAPs (PhAP71.1, - 50.3, - 43.2, and - 88.6) were specifically expressed in the leaves (Fig. 7). Further, 12 PhAPs (PhAP70.2, - 17.1, - 7.1, - 7.3, - 45.2, - 4.2, $93.5,-27.1,-40.1,-31.2,-31.3$ and 2.1$)$ were expressed in only the shoots, and three PhAPs (PhAP58.1, - 39.2 and -88.1) were expressed only in the rhizomes.

\section{Expression dynamics of PhAP genes in the rapid-growth stage of moso bamboo}

One of the most important features of moso bamboo is its rapid growth, which is mainly mediated by cell division and elongation [45]. Therefore, we further analyzed the expression patterns of the $129 \mathrm{PhAPs}$ in the top, middle and basal portions of $0.2 \mathrm{~m}, 1.5 \mathrm{~m}, 3 \mathrm{~m}$ and $6.7 \mathrm{~m}$ tall moso bamboo, as previously reported [43]. Figure 8 shows that the expression patterns of the PhAPs also showed tissue specificity. The percentages of highly expressed genes in different parts of the shoots are summarized in Figure S3B. The PhAPs were divided into two main classes (class I' and class II') based on their expression abundance (Fig. 8). The transcript reads were detected, except for those of class I' $\varepsilon$ (Fig. 8). All PhAP members of class I' were nearly absent from the $6.7 \mathrm{~m}$ moso bamboo (Fig. 8). Class I' $\alpha$ members were highly expressed in the top portion of 3 $\mathrm{m}$ moso bamboo, and their transcript levels gradually decreased in the middle and basal portions (Fig. 8). In contrast, class I' $\gamma$ transcripts accumulated in the basal portion of $3 \mathrm{~m}$ moso bamboo (Fig. 8), suggesting that the genes encoding those transcripts may be involved in cell elongation and SCW processes. Class I' $\delta$ transcripts preferentially accumulated in the top portion of the $0.2 \mathrm{~m}$ and $1.5 \mathrm{~m}$ moso bamboo shoots and in the middle portion of the $0.2 \mathrm{~m}$ moso bamboo shoots (Fig. 8 ); the genes encoding these transcripts may play critical roles in cell division. The mRNA level of class I' $\zeta$ members was slightly higher in the middle portion than in the other portions (Fig. 8). Compared with those of class I', PhAP members of class II' were expressed in $6.7 \mathrm{~m}$ moso bamboo (Fig. 8). For example, nearly all members from class II' $\alpha$ showed high mRNA abundance in the shoots of $6.7 \mathrm{~m}$ moso bamboo, although their expression was also detected in the basal portion of $0.2 \mathrm{~m}$ bamboo shoots (Fig. 8). Moreover, the members of class II' $\beta$ had a high transcript level in the top portion of the $0.2 \mathrm{~m}$ and $1.5 \mathrm{~m}$ shoots. Taken together, these results suggested that specific $P h A P$ s played a role in moso bamboo during different stages of the rapidgrowth period.

GA is considered to be one of the most important phytohormones involved in moso bamboo rapid growth, which includes cell expansion, SCW and PCD [44, 61]. APs have been reported to be involved in cell expansion and PCD processes and are associated with GA signaling $[28,62]$. Therefore, using previously reported RNA-seq data, we analyzed whether $P h A P$ s respond to GA treatment [56]. We further carried out RT-qPCR by randomly selecting seven genes to confirm the RNA-seq data. The leaves from two-month-old moso bamboo seedlings were treated with $100 \mu \mathrm{M} \mathrm{GA3}$; the RT-qPCR results were consistent with the RNA-seq results (Figure S4). After GA treatment, 39.51\% (32 PhAPs) and 43.21\% (35 PhAPs) of PhAP expression levels were positively and negatively regulated, respectively, by GA, while the expression of $17.28 \%$ (14 PhAPs) of the PhAPs was not detected, and others exhibited poor reproducibility (Fig. 9 and Figure S3C). Almost all GA-responsive PhAP genes were involved in the rapid growth of moso bamboo (Figure S3). These results suggested that the PhAP genes may respond to GA signaling to regulate various aspects of the rapid growth of moso bamboo. Altogether, these results strongly suggested that most $P h A P$ s play important roles in rapid growth. 


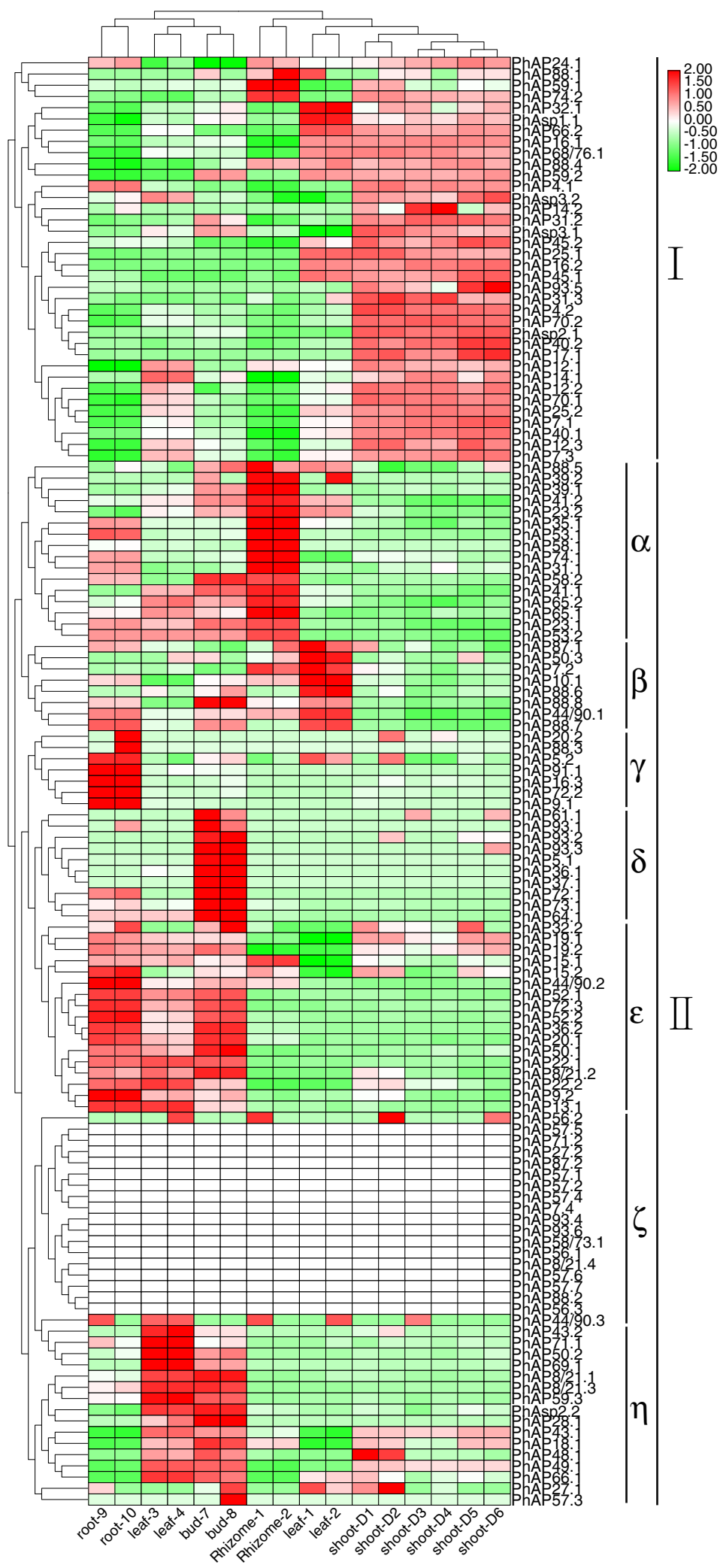

Fig. 7 (See legend on next page.) 
(See figure on previous page.)

Fig. 7 Expression patterns of PhAPs in different tissues of moso bamboo. The expression profiles of the 129 PhAPs is displayed by hierarchical clustering. The color bar in the upper-right corner indicates log2-based transcripts per million reads (TPM). Roots 9 and 10 represent 2 biological replicates of new roots with lateral roots, leaves 1 and 2 represent 2 biological replicates of leaf blades, leaves 3 and 4 represent 2 biological replicates of leaf sheaths, rhizomes 1 and 2 represent 2 replicates of rhizomes, and buds 1 and 2 represent 2 replicates of buds on rhizomes. Shoots D1 and D2, D3 and D4 and D5 and D6 represent 2 replicates of the top, middle and basal portions of the $6.7 \mathrm{~m}$ high shoot. The TPM value was listed in Table 54

\section{Discussion}

As a class of proteolytic enzymes, APs are involved in protein processing and degradation. Increasing amounts of evidence have demonstrated that plant APs play critical functions in plant development and in response to biotic and abiotic stresses. With the completion of whole-genome sequencing of a large number of plant species, members of AP gene families have been identified from additional plant species, such as Arabidopsis, poplar, rice and grape. However, little information is known about the $A P$ gene family in moso bamboo, which is one of the fastest growing plant species on Earth. In this study, we identified a total of 129 moso bamboo PhAPs that have conserved protein domains and gene structures and that are evolutionary related to members of the OsAP gene family. Expression pattern analysis also indicated that these PhAPs may be involved in the development of different organs and in rapid growth-related processes in moso bamboo.

APs were widely distributed across plant species, and we identified 129 PhAP genes from the moso bamboo genome, the number of which was greater than that of rice (96 OsAPs) [12], poplar (67 PtAPs) [22], Arabidopsis (69 AtAPs) [9], and grape (50 VqAPs) [21]. The 129 PhAP members could be classified into three categories: category A (16 PhAPs), category B (25 members), and category C (88 members) (Figs. 1, 2 and Table S1). Category A was the smallest category, and category $\mathrm{C}$ revealed gene expansion had occurred for its members, which was similar to findings in Arabidopsis, rice, poplar and grape $[9,12,21,22]$. Perhaps the formation of these three categories occurred before the monocotyledon and dicotyledon divergence. When they were compared with those from Arabidopsis, grape, poplar and rice, the PhAPs in moso bamboo revealed gene expansion had occurred for members in each category, especially categories A and B, which were approximately two times larger than they were in rice (Fig. 2). It has been reported that moso bamboo has experienced wholegenome duplication, which led to more gene duplication events [53]. We found that 56.6\% (73 PhAPs) of PhAPs were located in duplication blocks (Fig. 3), the percentage of which was greater than that in rice $(24.7 \%, 23$ $O s A P \mathrm{~s})$ [12] and grape $(32.0 \%, 16 V v A P \mathrm{~s})$ [21] but less than that in poplar $(62.6 \%, 42$ PtAPs $)$ [22]. In addition, we also identified tandem duplication events in the
PhAP family, such as PhAP8/21 s, PhAP57 and PhAP93 (Fig. 3). Category C of PhAPs also underwent gene expansion, as determined for PhAP57 and PhAP93, but some homologs of OsAPs, such as OsAP77-87, were lost during the evolutionary process (Fig. 3). These results suggested that, owing to the different development and growth features, compared with those in rice, APs in moso bamboo underwent a distinct evolutionary pathway. For example, moso bamboo exhibits rapid growth characteristics in the early growth stage $[43,45,59]$.

The protein domain of PhAPs was conserved during the evolutionary process. For example, all members contain a conserved full-length Asp domain with a catalytic sequence (Fig. 1); few PhAPs had no catalytic sequence, similar to those in rice and poplar [12, 22]. Signal peptides and transmembrane domains were also present in homologous genes of moso bamboo, such as those in rice, Arabidopsis, poplar and grape [9, 12, 21, 22] (Fig. 1). Motifs 1 and 2 contained the catalytic sequence, and motifs 4 and 9 were conserved and present in nearly all members of the three categories (Figs. 4 and 5), which is similar to that described in poplar [22]. Members of each category shared the conserved motif (Fig. 4), and the members of each category contained a specific motif, for example, motif 8 , which was category A specific, and motifs $3,6,7$ and 10 , which were category $B$ and $C$ specific (Fig. 4). These results suggested that motifs of the PhAP family are diverse. The gene structures of PhAPs from the same category were more conserved than those from different categories (Fig. 4). The gene structure of the members of category $\mathrm{B}$ was most conserved, while that of category A and category $\mathrm{C}$ was less conserved and was similar to the gene structure of the corresponding members of category $\mathrm{A}$ and category $\mathrm{C}$ in grape and poplar but different from those in rice [12, 21, 22]. These results suggested that PhAPs may have originated from an ancient DNA sequence and experienced expansion with variation. The domain varied among the three categories, although the Asp domain and catalytic sequence were conserved, suggesting that there may be functional divergence.

Gene expression is specifically controlled in space and time, and the precise regulation by developmental and environmental signals are essential for gene function. AP genes are reported to be widely expressed in plants [9, 12, 21, 22]. PhAPs had high transcript abundance in 


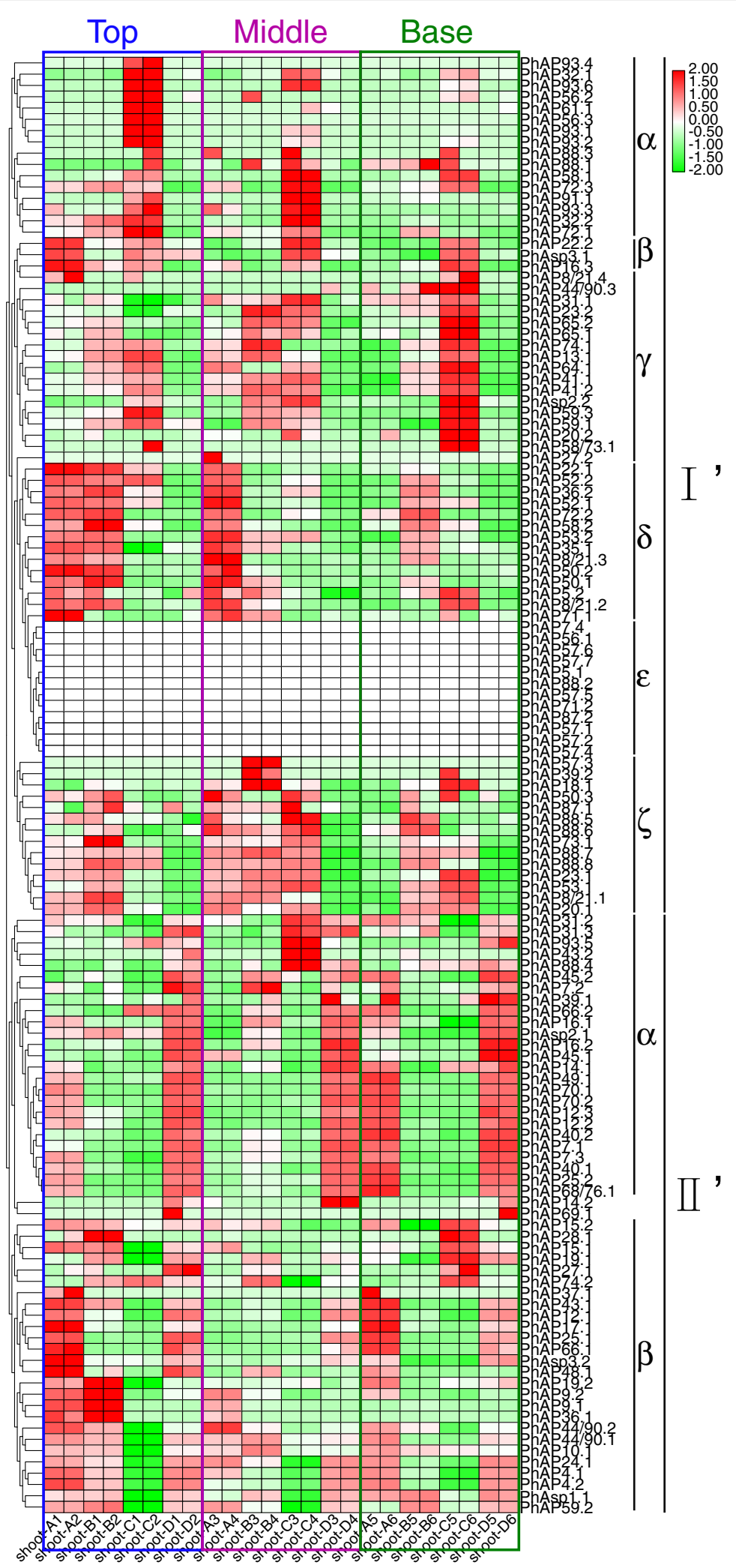

Fig. 8 (See legend on next page.) 
(See figure on previous page.)

Fig. 8 Expression heatmap of PhAPs in the top, middle and basal portions of rapidly growing shoots of moso bamboo. The expression profile of 129 PhAPs is displayed by hierarchical clustering. The color bar in the upper-right corner indicates log2-based TPM values. Shoots A, B, C, and D represent the basal portions of $0.2 \mathrm{~m}, 1.5 \mathrm{~m}, 3 \mathrm{~m}$ and $6.7 \mathrm{~m}$ high rapidly growing moso bamboo, respectively. The numerals 1 and 2, 3 and 4, and 5 and 6 represent 2 biological replications. The TPM was listed in Table S5

nearly all tissues, including the roots, leaves, buds, shoots and rhizomes, although no transcripts were detected for a few PhAPs (Fig. 7). Some PhAPs also exhibited tissue-specific expression patterns; for example, four $P h A P s$ and three PhAPs were specifically highly expressed in the roots and rhizomes, respectively (Fig. 7). These specific expression patterns were similar to those in other plant species. For example, three AP genes were not detected in the tested tissues, while other APs were generally expressed in root leaves, stems, flowers, fruits and tendrils with various abundances in grape [21]. In poplar, AP genes exhibited preferential expression patterns in the mature and young leaves, roots, female and male catkins and xylem [22]. In addition, ciselements reported to respond to environmental change, phytohormones, PCD and SCW were located in the promoter region of APs in moso bamboo (Fig. 6 and Fig. 1). These results indicated that AP genes may function in various tissues in moso bamboo and are potentially regulated by various developmental and environmental cues. The mechanism of PhAP tissue-specific expression patterns and the contribution of various factors is an interesting question worthy of further study.

Rapid growth of moso bamboo was observed shortly after the young shoots emerged from the ground [43]. GA is a critical phytohormone involved in rapid growth, and the spatial and temporal distribution of GA is strictly regulated $[44,61]$. The top portion of young bamboo shoots contain GA at a concentration higher than that in the basal portion [45]. Pith cavity formation accompanied by PCD and cell division are the main biological events in the top portion during the moso bamboo rapid-growth stage $[44,45]$. We found that 59 PhAPs were expressed in the top region of the $0.2 \mathrm{~m}$ moso bamboo shoots (Fig. 8). Among them, PhAP25s, which is the ortholog of OsAP25 in rice and is triggered by PCD in plants [35], was highly expressed at the top and was expressed at low levels in the middle or basal part (Fig. 8). Notably, PhAP25s was activated by GA treatment (Fig. 9). These results suggest that PhAP25s may be regulated by GA and may play a role in pith cavity formation in the early stage of rapid bamboo growth. In the middle and basal portions, cell elongation coupled with cell wall thickening is fundamental [45]. PhAP members from class I' $\alpha$, class I' $\gamma$ and class II' $\alpha$ were highly expressed in the middle and basal portions (Fig. 8). Among them, the mRNA abundance of PhAP65.1, PhAP65.2 and PhAP19.1 peaked in the basal portion of the $3 \mathrm{~m}$ moso bamboo shoots (Fig. 8). In rice, OsAP65 is involved in pollen germination and pollen tube growth [32]. The homologs of PhAP19.1 in Arabidopsis were $A 36$ and $A 39$, which encode two aspartic proteases that are preferentially transcribed in pollen and that affect pollen tubes and tube cell wall deposition [18]. These results suggested that PhAPs highly expressed in the middle and basal portions may play functions in cell elongation and cell wall component formation. It is interesting that not all APs highly expressed in the top part were activated by GA or that those highly expressed in the basal part were repressed by GA. One reason is that GA is not the unique regulatory factor of PhAPs; these genes may be regulated by other developmental and environmental signaling pathways. How PhAP expression is precisely controlled requires further investigation.

Because the shell of bamboo covers the stem of young shoots, which presented a yellow-like etiolation status $[43,44]$, the rapid growth of moso bamboo was similar to skotomorphogenesis, including protein degradation processes [63]. We found that light-responsive elements were located in nearly all PhAP upstream promoter regions (Fig. 6). It was reported that the A1 expression level in Arabidopsis is upregulated by light [64]. We also found that nearly all PhAP expression levels were dynamic at different stages (Fig. 8). The phytohormone GA has been reported to be involved in skotomorphogenesis, and the GA concentration reached its maximum in the basal portion of moso bamboo [45, 65]. Most PhAPs also positively or negatively respond to GA treatment (Fig. 9), although a small amount of GA-responsive cis-elements were identified in the promoter region of the PhAPs (Fig. 6). Most PhAPs may not be directly regulated by GA during the moso bamboo rapid-growth stage. Expression of APs regulated by GA was previously reported in Arabidopsis; for example, ASPG1, which is critical for seed germination, is activated by GA treatment [28]. This indicated that PhAPs involved in rapid growth are activated to participate cell division or elongation during the different stages. Altogether, these results strongly suggest that PhAPs play critical roles in rapid growth of moso bamboo by integrating developmental and environmental signaling.

\section{Conclusion}

Aspartic proteases are important proteolytic enzymes that function in plant development and in response to 


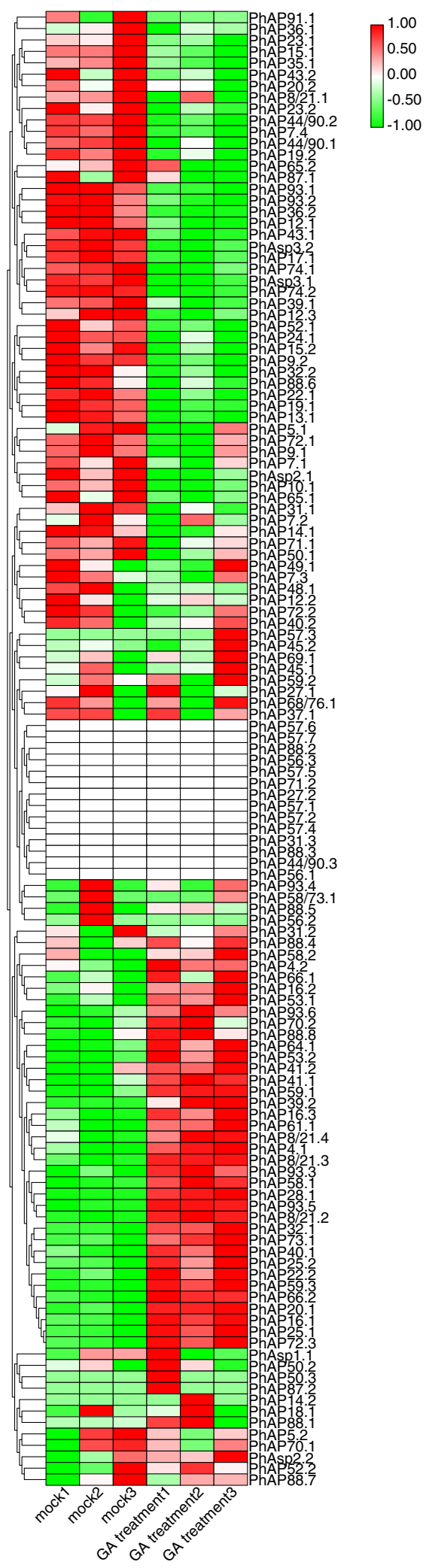

Fig. 9 Expression heatmap of PhAPs in response to GA treatment. The expression profiles of the 129 PhAPs in response to GA (100 $\mu \mathrm{M}$ for $4 \mathrm{~h}$ ) are displayed by hierarchical clustering. The color bar in the upper-right corner indicates log2-based expression values. The TPM was listed in Table $\mathbf{S 6}$

environmental changes. In this study, a total of 129 PhAPs were identified from moso bamboo, a fastgrowing plant species. The PhAP family in moso bamboo underwent gene expansion via segmental and tandem duplications that were distinct from those in rice. Notably, several sets of PhAP genes showed dynamic transcript abundance during the moso bamboo rapidgrowth stage, suggesting that PhAPs may play critical roles in moso bamboo rapid growth by mediating environmental and developmental signaling.

\section{Methods}

\section{Genome resources}

Chromosome-level reference genomes of moso bamboo (Phyllostachys edulis) and whole rice (Oryza sativa) plants were downloaded from the GigaDB Database [43] (http://gigadb.org/dataset/100498) and Phytozome database (https://genome.jgi.doe.gov/portal/pages/dynamic OrganismDownload.jsf?organism=Osativa), respectively.

\section{Identification of members of the asp gene family}

To identify moso bamboo Asp genes, predicted proteins from the moso bamboo genomic database were searched by HMMER v3 [66] using the hidden Markov model (HMM) file that corresponded to the Asp domain (PF00026) downloaded from the Pfam database (http:// pfam.xfam.org/) as a query [67]. The obtained protein sequences with an expected value $(\mathrm{E})<1 \mathrm{E}-20$ and containing the Asp domain were aligned by ClustalW [68] and used to construct a moso bamboo-specific HMM file via hmmbuild from HMMER v3. The new moso bamboo-specific Asp HMM was used as a query against the predicted proteins of moso bamboo. All peptide sequences with $\mathrm{E}<1 \mathrm{E}-2$ and containing the Asp domain identified by the Pfam database [67] and NCBI-CDD tools [69] were selected as Asp genes (Table S1). OsAP genes were reported previously [12], we confirmed them in Phytozome database and listed in Table S2.

\section{Multiple sequence alignment and phylogenetic and domain analyses}

$129 \mathrm{PhAPs}$ and 92 OsAPs amino acid residues were aligned by MEGA 7.0. The alignment files were uploaded to the IQ-TREE web server [54] (http://iqtree. cibiv.univie.ac.at/) for phylogenetic tree construction, with the default parameters. The protein domains were identified by searching the Pfam database. EvolView [70-72] (https://www.evolgenius.info/evolview/\#login) 
and Dendscope 3 [73, 74] were used for phylogenetic tree visualization and annotations.

\section{Protein motif and gene structure analyses}

The conserved motifs of the PhAP genes were identified by the MEME Suite web server [55] (http://meme-suite. org/). The number of motifs was set to 10 , and all other parameters were the default ones. The gene structure and conserved domain were visualized via TBtools [75].

\section{Chromosomal distribution and gene duplication}

The length of the chromosomes and the locations of the $\mathrm{PhAP}$ genes in the moso bamboo genome were used to map genes onto the chromosomes through MapChart [76]. Nucleotide sequences with alignment ratios and similarity ratios greater than $70 \%$ and with distances between genes on the same chromosome of less than 100 $\mathrm{kb}$ were selected as tandem duplications. Moreover, genes located in duplication regions and nucleotide sequences with alignment ratios greater than $75 \%$ were selected as resulting from segmental duplications [77-80]. The $K \mathrm{a} / \mathrm{Ks}$ values were calculated by TBtools [75]. The formula $\mathrm{T}=K \mathrm{~s} / 2 \mathrm{r}$ was used to estimate the divergence time of PhAP gene pairs in moso bamboo. The $r$ for moso bamboo and rice was $6.5 \times 10^{-9}$ years [81]. A diagram of collinearity analysis of genes and chromosomes was constructed using Circos [82].

\section{Cis-element analysis}

The 1500 bp upstream DNA sequence of the $5^{\prime}$-UTR of the PhAP genes was selected as the promoter sequence. The promoter sequences were uploaded to the PlantCARE database (http://bioinformatics.psb.ugent.be/webtools/ plantcare/html/) to scan for cis-elements. The cis-elements from the PlantCARE database were subsequently screened manually. The identification and location of previously reported $\mathrm{PCD}$ - and SCW-related cis-elements (SNBE, TERE, M46RE, ACI, ACII, ACIII, SMRE1, SMRE2, SMRE3 and SMRE5) were scanned via the MEME Suite web server [55].

\section{Expression analysis of $P h A P$ genes}

The raw RNA-seq data for new roots with lateral roots; blades; leaf sheaths; buds on rhizomes; rhizomes; and top, middle and basal portions of bamboo shoots of different heights $(0.2 \mathrm{~m}, 1.5 \mathrm{~m}, 3 \mathrm{~m}$ and $6.7 \mathrm{~m})$ were retrieved from the GigaDB dataset [43]. The raw RNA-seq data for GA treatment were retrieved from NCBI Sequence Read Archive (SRA) under the accession number GSE104596. Quantification of transcript expression was carried out by Salmon [83], and TPM were obtained for further analysis.

\section{RT-qPCR analysis}

Moso bamboo tissue materials (top, middle and basal portions of the shoots, leaf blades, sheaths and roots) were harvested from $8 \mathrm{~m}$-high bamboo growing in a bamboo forest at Fujian Agriculture and Forestry University (FAFU). Two-month-old seedlings were treated with $100 \mu \mathrm{M}$ GA3 for $4 \mathrm{~h}$ [61]. The control groups were treated with the same concentration of DMSO instead of $100 \mu \mathrm{M}$ GA3 for $4 \mathrm{~h}$. Samples (three independent replications) were collected, immediately frozen in liquid nitrogen and stored at $-80^{\circ} \mathrm{C}$.

Total RNA of the samples was extracted using an RNAprep Pure Plant Plus Kit (Tiangen, China). Total RNA was then reverse transcribed using a PrimeScript RT Reagent Kit together with gDNA Eraser (Takara, China). RT-qPCR was performed via GoTaq qPCR Master Mix (Promega, USA). $P h U B Q$ was used as an internal control gene [61]. All the primers used in this study are listed in Table S7. The reaction mixture consisted of $10 \mu \mathrm{l}$ of $2 \times$ GoTaq qPCR Master Mix, $0.4 \mu \mathrm{l}$ of each gene-specific primer, $1 \mu \mathrm{l}$ of cDNA and $8.2 \mu \mathrm{l}$ of nuclease-free water. The reaction conditions were as follows: $95^{\circ} \mathrm{C}$ for 2 min followed by 40 cycles of $95^{\circ} \mathrm{C}$ for $15 \mathrm{~s}$ and $60^{\circ} \mathrm{C}$ for $20 \mathrm{~s}$. The relative gene expression levels were calculated by the comparative $\Delta \mathrm{Ct}$ method $\left(2^{-\Delta \mathrm{Ct}}\right)$. Three biological replications were assessed per sample.

\section{Supplementary Information}

The online version contains supplementary material available at https://doi. org/10.1186/s12864-020-07290-7.

Additional file 1: Table S1. The information of AP family genes in Phyllostachys edulis.

Additional file 2: Table S2. The information of AP family genes in Oryza sativa.

Additional file 3: Table S3. Estimated divergence time of AP gene pairs in P. edulis.

Additional file 4: Figure S1. Analysis of PCD- and SCW-related cis-elements in the PhAP promoters.

Additional file 5: Figure S2. Expression level of seven selected PhAPS in different tissues of moso bamboo.

Additional file 6: Figure S3. Percentage of PhAPs highly expressed in different tissues, in response to GA treatment and at different developmental periods of shoots.

Additional file 7: Figure S4. Expression level of seven selected PhAPS after GA treatment.

Additional file 8: Table S4. The expression data of PhAPs in different tissues.

Additional file 9: Table S5. The expression data of PhAPs at different developmental periods of moso bamboo shoot.

Additional file 10: Table S6. The expression data of PhAPs under GA treatment.

Additional file 11: Table S7. RT-qPCR primers used for PhAP genes.

Abbreviations

AP: Aspartic protease; APs: Aspartic proteases; PCD: Programmed cell death; ASPG1: ASPARTIC PROTEASE IN GUARD CELL 1; CDR1: CONSTITUTIVE DISEASE RESISTANCE 1; ASPR1: ATYPICAL ASPARTIC PROTEASE IN ROOT 1; EAT1: ETER 
NAL TAPETUM 1; PCS1: PROMOTION OF CELL SURVIVAL; SCW: Secondary cell wall; TPM: Transcripts per million reads

\section{Acknowledgements \\ Not applicable.}

\section{Authors' contributions}

B.L. and J.Z. conceived the project. X.W., X.Y. and L.G. collected the public data, carried out the bioinformatics analysis, and interpreted the data. S.L., S.Z., and Y. J worked for bamboo sample collection, RT-qPCR experiment and data analysis. X. W. wrote the manuscript draft. B.L., J.Z., S.Z. and Y. J. revised the manuscript. All authors have read and approved the manuscript.

\section{Funding}

This study was funded by the Forestry Peak Discipline Construction Project (71201800721) and the National Natural Science Foundation of China (31870661) to B.L., and the Science and Technology Innovation Special Fund at Fujian Agriculture and Forestry University (CXZX2019044G) to B.L. and S.Z. J.Z. is funded by the Zhejiang A\&F University Research and Development Fund Talent Startup Project. The funder had no role in study design, data collection and analysis, data interpretation or preparation of the manuscript.

\section{Availability of data and materials}

All data supporting the conclusions of this article are provided within the article and its additional files. The genomics sequences data of moso bamboo and rice are available in the GigaDB Database (http://gigadb.org/ dataset/100498) and Phytozome database (https://genome.jgi.doe.gov/ portal/pages/dynamicOrganismDownload.jsf?organism=Osativa), respectively. The public RNA-seq data are available on GigaDB Database (http://gigadb. org/dataset/100498) and NCBI GEO under the accession number GSE104596.

\section{Ethics approval and consent to participate}

Not applicable.

\section{Consent for publication}

Not applicable.

\section{Competing interests}

The authors declare no conflict of interest.

\section{Author details}

${ }^{1}$ College of Forestry, Fujian Agriculture and Forestry University, Fuzhou 350002, China. ${ }^{2}$ Fujian Colleges and Universities Engineering Research Institute of Conservation \& Utilization of Natural Bioresources, Fujian Agriculture and Forestry University, Fuzhou 350002, China. ${ }^{3}$ State Key Laboratory of Subtropical Silviculture, School of Forestry and Biotechnology, Zhejiang A\&F University, Zhejiang 311300, Hangzhou, China.

Received: 11 February 2020 Accepted: 28 November 2020 Published online: 10 January 2021

\section{References}

1. Simoes I, Faro C. Structure and function of plant aspartic proteinases. Eur J Biochem. 2004;271(11):2067-75.

2. Mutlu A, Gal S. Plant aspartic proteinases: enzymes on the way to a function. Physiol Plant. 1999;105:569-76.

3. Davies DR. The structure and function of the aspartic proteinases. Annu Rev Biophys Biophys Chern. 1990;19:189-215.

4. Dunn BM. Structure and mechanism of the pepsin-like family of aspartic peptidases. ChemRev. 2002;102(12):4431-58.

5. Rawlings ND, Barrett AJ. Families of aspartic peptidases, and those of unknown catalytic mechanism. Methods Enzymol. 1995;248:105-20.

6. Rawlings ND, Barrett AJ. MEROPS: the peptidase database. Nucleic Acids Res. 1999;27(1):325-31

7. Mandujano-Gonzalez V, Tellez-Jurado A, Anducho-Reyes MA, Arana-Cuenca A, Mercado-Flores Y. Purification and characterization of the extracellular aspartyl protease APSm1 from the phytopathogen fungus Stenocarpella maydis. Protein Expr Purif. 2016;117:1-5.

8. Cooper JB. Aspartic proteinases in disease: a structural perspective. Curr Drug Targets. 2002;3(2):155-73.
9. aSG CF. Aspartic proteinase content of the Arabidopsis genome. Curr Protein Pept Sci. 2005;6:493-500.

10. Park H, Yamanaka N, Mikkonen A, Kusakabe I, Kobayashi H. Purification and characterization of aspartic proteinase from sunflower seeds. Biosci Biotechnol Biochem. 2000;64(5):931-9.

11. Chen F, Foolad MR. Molecular organization of a gene in barley which encodes a protein similar to aspartic protease and its specific expression in nucellar cells during degeneration. Plant Mol Biol. 1997;35:821-31.

12. Chen J, Ouyang Y, Wang L, Xie W, Zhang Q. Aspartic proteases gene family in rice: gene structure and expression, predicted protein features and phylogenetic relation. Gene. 2009;442(1-2):108-18.

13. Timotijevic GS, Radovic SR, Maksimovic VR. Characterization of an aspartic proteinase activity in buckwheat (Fagopyrum esculentum Moench) seeds. J Agric Food Chem. 2003;51(7):2100-4.

14. Belozersky MA, Sarbakanova ST, Dunaevsky YE. Aspartic proteinase from wheat seeds: isolation, properties and action on gliadin. Planta. 1989;177: 321-6.

15. An Cl, Fukusaki E, Kobayashi A. Aspartic proteinases are expressed in pitchers of the carnivorous plant Nepenthes alata Blanco. Planta. 2002; 214(5):661-7.

16. Faro C, Ramalho-Santos M, Vieira M, Mendes A, Simões I, Andrade R, et al. Cloning and characterization of cDNA encoding Cardosin a, an RGDcontaining plant aspartic proteinase. J Biol Chem. 1999;274(40):28724-9.

17. Yao X, Xiong W, Ye T, Wu Y. Overexpression of the aspartic protease ASPG1 gene confers drought avoidance in Arabidopsis. J Exp Bot. 2012;63(7):257993.

18. Gao H, Li R, Guo Y. Arabidopsis aspartic proteases A36 and A39 play roles in plant reproduction. Plant Signal Behav. 2017;12(4):e1304343.

19. Guo R, Tu M, Wang X, Zhao J, Wan R, Li Z, et al. Ectopic expression of a grape aspartic protease gene, AP13, in Arabidopsis thaliana improves resistance to powdery mildew but increases susceptibility to Botrytis cinerea. Plant Sci. 2016;248:17-27.

20. Prasad BD, Creissen G, Lamb C, Chattoo BB. Heterologous expression and characterization of recombinant OsCDR1, a rice aspartic proteinase involved in disease resistance. Protein Expr Purif. 2010;72(2):169-74.

21. Guo R, Carole B, Li X, Gao M, Zheng Y, Wang X. Genome-wide idetification, evolutionary and expression analysis of the aspartic protease gene suprefamily in grape. BMC Genomics. 2013;14:554.

22. Cao S, Guo M, Wang C, Xu W, Shi T, Tong G, et al. Genome-wide characterization of aspartic protease (AP) gene family in Populus trichocarpa and identification of the potential PtAPs involved in wood formation. BMC Plant Biol. 2019;19(1):276.

23. Glathe S, Kervinen J, Nimtz M, Li GH, Tobin GJ, Copeland TD, et al. Transport and activation of the vacuolar aspartic proteinase Phytepsin in barley (Hordeum vulgare L.). J Biol Chem. 1998;273(47):31230-6.

24. Asakura T, Abe K, Arai S. Evidence for the occurrence of multiple aspartic proteinases in rice seeds. Biosci Biotechnol Biochem. 1995;59(9):1793-4.

25. Kathleen D'HSOI, Bosch D, Van Damme J, Goethals M, Vandekerckhove J. Krebbers E. An aspartic proteinase present in seeds cleaves albumin precursors in vitro. J Biol Chem. 1993;268:20884-91.

26. Asakura T, Watanabe H, Abe K, Arai S. Rice aspartic proteinase, oryzasin, expressed during seed ripening and germination, has a gene organization distinct from those of animal and microbial aspartic proteinases. Eur J Biochem. 1995;232(1):77-83.

27. Tamura T, Terauchi K, Kiyosaki T, Asakura T, Funaki J, Matsumoto I, et al. Differential expression of wheat aspartic proteinases, WAP1 and WAP2, in germinating and maturing seeds. J Plant Physiol. 2007;164(4):470-7.

28. Shen W, Yao X, Ye T, Ma S, Liu X, Yin X, et al. Arabidopsis aspartic protease ASPG1 affects seed dormancy, seed longevity and seed germination. Plant Cell Physiol. 2018;59(7):1415-31.

29. TSkes ZA. Digestive enzymes secreted by the carnivorous plant nepenthes macferlanei L. Planta. 1974;119:39-46.

30. Xia Y, Suzuki H, Borevitz J, Blount J, Guo Z, Patel K, et al. An extracellular aspartic protease functions in Arabidopsis disease resistance signaling. EMBO J. 2004;23:980-8.

31. Prasad BD, Creissen G, Lamb C, Chattoo BB. Overexpression of Rice (Oryza sativa L.) OsCDR1 leads to Constitutive activation of defense responses in Rice and Arabidopsis. MPMI. 2009;22(12):1635-44.

32. Huang J, Zhao X, Cheng K, Jiang Y, Ouyang Y, Xu C, et al. OsAP65, a rice aspartic protease, is essential for male fertility and plays a role in pollen germination and pollen tube growth. J Exp Bot. 2013;64(11):3351-60. 
33. Soares A, Niedermaier S, Faro R, Loos A, Manadas B, Faro C, et al. An atypical aspartic protease modulates lateral root development in Arabidopsis thaliana. J Exp Bot. 2019;70(7):2157-71.

34. Runeberg-Roos P, Saarma M. Phytepsin, a barley vacuolar aspartic proteinase, is highly expressed during autolysis of developing tracheary elements and sieve cells. Plant J. 1998;15(1):139-45.

35. Niu N, Liang W, Yang $X$, Jin W, Wilson ZA, Hu J, et al. EAT1 promotes tapetal cell death by regulating aspartic proteases during male reproductive development in rice. Nat Commun. 2013;4:1445.

36. Ge X, Dietrich C, Matsuno M, Li G, Berg H, Xia Y. An Arabidopsis aspartic protease functions as an anti-cell-death component in reproduction and embryogenesis. EMBO Rep. 2005;6(3):282-8.

37. Pyo H, Demura T, Fukuda H. TERE; a novel cis-element responsible for a coordinated expression of genes related to programmed cell death and secondary wall formation during differentiation of tracheary elements. Plant J. 2007;51(6):955-65.

38. Zhong $\mathrm{R}$, Lee $\mathrm{C}$, Ye Z $\mathrm{H}$. Global analysis of direct targets of secondary wall NAC master switches in Arabidopsis. Mol Plant. 2010;3(6):1087-103.

39. Zhong R, Ye ZH. MYB46 and MYB83 bind to the SMRE sites and directly activate a suite of transcription factors and secondary wall biosynthetic genes. Plant Cell Physiol. 2012;53(2):368-80.

40. Zhong R, McCarthy RL, Haghighat M, Ye ZH. The poplar MYB master switches bind to the SMRE site and activate the secondary wall biosynthetic program during wood formation. PLoS One. 2013;8(7):e69219.

41. Ohashi-lto K, Oda Y, Fukuda H. Arabidopsis VASCULAR-RELATED NAC-DOMAIN6 directly regulates the genes that govern programmed cell death and secondary wall formation during xylem differentiation. Plant Cell. 2010;22(10):3461-73.

42. Zhao H, Zhao S, International Network for B, Rattan FB, Liu H, Yang H, et al. Announcing the genome atlas of bamboo and rattan (GABR) project: promoting research in evolution and in economically and ecologically beneficial plants. Giga Sci. 2017;6(7):1-7.

43. Zhao H, Gao Z, Wang L, Wang J, Wang S, Fei B, et al. Chromosome-level reference genome and alternative splicing atlas of moso bamboo (Phyllostachys edulis). Giga Sci. 2018;7(10):1-12.

44. Guo L, Sun X, Li Z, Wang Y, Fei Z, Jiao C, et al. Morphological dissection and cellular and transcriptome characterizations of bamboo pith cavity formation reveal a pivotal role of genes related to programmed cell death Plant Biotechnol J. 2019;17(5):982-97.

45. Cui K, He CY, Zhang JG, Duan AG, Zeng YF. Temporal and spatial profiling of internode elongation-associated protein expression in rapidly growing culms of bamboo. J Proteome Res. 2012;11(4):2492-507.

46. Li L, Cheng Z, Ma Y, Bai Q, Li X, Cao Z, et al. The association of hormone signalling genes, transcription and changes in shoot anatomy during moso bamboo growth. Plant Biotechnol J. 2018;16(1):72-85.

47. Shan, Yang, Xu, Zhu, Gao. Genome-wide investigation of the NAC gene family and its potential association with the secondary Cell Wall in Moso bamboo. Biomolecules. 2019;9(10):609.

48. Li L, Yang K, Wang S, Lou Y, Zhu C, Gao Z. Genome-wide analysis of laccase genes in moso bamboo highlights PeLAC10 involved in lignin biosynthesis and in response to abiotic stresses. Plant Cell Rep. 2020;39(6):751-63.

49. Hou D, Cheng Z, Xie L, Li X, Li J, Mu S, et al. The R2R3MYB gene family in Phyllostachys edulis: genome-wide analysis and identification of stress or development-related R2R3MYBs. Front Plant Sci. 2018;9:738.

50. Biswas P, Chakraborty S, Dutta S, Pal A, Das M. Bamboo flowering from the perspective of comparative genomics and Transcriptomics. Front Plant Sci. 2016;7:1900

51. Dutta S, Biswas P, Chakraborty S, Mitra D, Pal A, Das M. Identification characterization and gene expression analyses of important flowering genes related to photoperiodic pathway in bamboo. BMC Genomics. 2018;19(1):190.

52. Chiu WB, Lin CH, Chang CJ, Hsieh MH, Wang AY. Molecular characterization and expression of four CDNAs encoding sucrose synthase from green bamboo Bambusa oldhamii. New Phytol. 2006;170(1):53-63.

53. Peng Z, Lu Y, Li L, Zhao Q, Feng Q, Gao Z, et al. The draft genome of the fast-growing non-timber forest species moso bamboo (Phyllostachys heterocycla). Nat Genet. 2013;45(4):456-61 461e451-2.

54. Trifinopoulos J, Nguyen LT, von Haeseler A, Minh BQ. W-IQ-TREE: a fast online phylogenetic tool for maximum likelihood analysis. Nucleic Acids Res. 2016:44(W1):W232-5.

55. Bailey TL, Boden M, Buske FA, Frith M, Grant CE, Clementi L, et al. MEME SUITE: tools for motif discovery and searching. Nucleic Acids Res. 2009;37: W202-8.
56. Ye ZH. MYB46 and MYB83 bind to the SMRE sites and directly activate a suite of transcription factors and secondary wall Biosyntheticn genes. Plant Cell Physiol. 2012;53(2):368-80.

57. Kim WC, Ko JH, Han $\mathrm{KH}$. Identification of a cis -acting regulatory motif recognized by MYB46, a master transcriptional regulator of secondary wall biosynthesis. Microbiology. 2012;78(4):489-501.

58. Daneva A, Gao Z, Van Durme M, Nowack MK. Functions and regulation of programmed cell death in plant development. Annu Rev Cell Dev Biol. 2016;32:441-68.

59. Zhang L, Xing D. Methyl Jasmonate induces production of reactive oxygen species and alterations in mitochondrial dynamics that precede photosynthetic dysfunction and subsequent cell death. Plant Cell Physiol. 2008:49(7):1092-111.

60. Kapoor K, Mira MM, Ayele BT, Nguyen TN, Hill RD, Stasolla C. Phytoglobins regulate nitric oxide-dependent abscisic acid synthesis and ethyleneinduced program cell death in developing maize somatic embryos. Planta. 2018;247(6):1277-91

61. Zhang H, Wang H, Zhu Q, Gao Y, Wang H, Zhao L, et al. Transcriptome characterization of moso bamboo (Phyllostachys edulis) seedlings in response to exogenous gibberellin applications. BMC Plant Biol. 2018;18(1): 125.

62. Morris K, Linkies A, Müller K, Oracz K, Wang X, Lynn JR, et al. Regulation of seed germination in the close Arabidopsis relative (Lepidium sativum): a global tissue-specific transcript analysis. Plant Physiol. 2011;155(4):1851-70.

63. Beers EP, Jones AM, Dickerman AW. The S8 serine, C1A cysteine and A1 aspartic protease families in Arabidopsis. Phytochemistry. 2004;65(1):43-58.

64. Chen X, Pfeil JE, Gal S. The three typical aspartic proteinase genes of Arabidopsis thaliana are differentially expressed. Eur J Biochem. 2002; 269(18):4675-84.

65. Ye S, Chen G, Kohnen MV, Wang W, Cai C, Ding W, et al. Robust CRISPR/ Cas 9 mediated genome editing and its application in manipulating plant height in the first generation of hexaploid Ma bamboo (Dendrocalamus latiflorus Munro). Plant Biotechnol J. 2020;18(7):1501-3.

66. Eddy SR. Multiple alignment using hidden Markov models. Proc Int Conf Intell Syst Mol Biol. 1995;3:114-20.

67. El-Gebali S, Mistry J, Bateman A, Eddy SR, Luciani A, Potter SC, et al. The Pfam protein families database in 2019. Nucleic Acids Res. 2019;47(D1):D427-32.

68. Larkin MA, Blackshields G, Brown NP, Chenna R, McGettigan PA, McWilliam H, et al. Clustal W and Clustal X version 2.0. Bioinformatics. 2007;23(21):2947-8.

69. Marchler-Bauer A, Derbyshire MK, Gonzales NR, Lu S, Chitsaz F, Geer LY, et al. CDD: NCBI's conserved domain database. Nucleic Acids Res. 2015; 43(Database issue):D222-6.

70. He Z, Zhang H, Gao S, Lercher MJ, Chen WH, Hu S. Evolview v2: an online visualization and management tool for customized and annotated phylogenetic trees. Nucleic Acids Res. 2016;44(W1):W236-41.

71. Subramanian B, Gao S, Lercher MJ, Hu S, Chen WH. Evolview v3: a webserver for visualization, annotation, and management of phylogenetic trees. Nucleic Acids Res. 2019;47(W1):W270-5.

72. Zhang H, Gao S, Lercher MJ, Hu S, Chen WH. EvolView, an online tool for visualizing, annotating and managing phylogenetic trees. Nucleic Acids Res. 2012;40:W569-72.

73. Huson DH, Richter DC, Rausch C, Dezulian T, Franz M, Rupp R. Dendroscope: An interactive viewer for large phylogenetic trees. BMC Bioinformatics. 2007; 8:460.

74. Huson DH, Scornavacca C. Dendroscope 3: an interactive tool for rooted phylogenetic trees and networks. Syst Biol. 2012;61(6):1061-7.

75. Chen C, Chen H, Zhang Y, Thomas HR, Frank MH, He Y, et al. TBtools: An integrative toolkit developed for interactive analyses of big biological data. Mol Plant. 2020;13(8):1194-202.

76. Voorrips RE. MapChart: software for the graphical presentation of linkage maps and QTLs. J Heredity. 2002;93(1):93-4.

77. Zhu Y, Wu N, Song W, Yin G, Qin Y, Yan Y, et al. Soybean (Glycine max) expansin gene superfamily origins: segmental and tandem duplication events followed by divergent selection among subfamilies. BMC Plant Biol. 2014;14:93.

78. Cannon SB, Mitra A, Baumgarten A, Young ND, May G. The roles of segmental and tandem gene duplication in the evolution of large gene families in Arabidopsis thaliana. BMC Plant Biol. 2004;4:10.

79. Xie T, Chen C, Li C, Liu J, Liu C, He Y. Genome-wide investigation of WRKY gene family in pineapple: evolution and expression profiles during development and stress. BMC Genomics. 2018;19(1):490. 
80. Vatansever R, Koc I, Ozyigit SU II, Uras ME, Anjum NA, Pereira E, et al. Genomewide identification and expression analysis of sulfate transporter (SULTR) genes in potato (Solanum tuberosum L.). Planta. 2016;244(6):1167-83.

81. Li L, Mu S, Cheng Z, Cheng Y, Zhang Y, Miao Y, et al. Characterization and expression analysis of the WRKY gene family in moso bamboo. Sci Rep. 2017;7(1):6675

82. Krzywinski M, Schein J, Birol I, Connors J, Gascoyne R, Horsman D, et al. Circos: an information aesthetic for comparative genomics. Genome Res. 2009;19(9):1639-45.

83. Patro R, Duggal G, Love MI, Irizarry RA, Kingsford C. Salmon provides fast and bias-aware quantification of transcript expression. Nat Methods. 2017; 14(4):417-9.

\section{Publisher's Note}

Springer Nature remains neutral with regard to jurisdictional claims in published maps and institutional affiliations.

Ready to submit your research? Choose BMC and benefit from:

- fast, convenient online submission

- thorough peer review by experienced researchers in your field

- rapid publication on acceptance

- support for research data, including large and complex data types

- gold Open Access which fosters wider collaboration and increased citations

- maximum visibility for your research: over $100 \mathrm{M}$ website views per year

At BMC, research is always in progress.

Learn more biomedcentral.com/submissions 